\section{Überleben und Todesursachen nach palliativer endobronchialer Brachytherapie mit Iridium 192-High dose bei rezidivierendem Bronchialkarzinom}

\author{
Eine Matched Pair Studie
}

\author{
H.-N. Macha' \\ P. Bach ${ }^{2}$ \\ B. Wahlers ${ }^{2}$ \\ G. Reichle ${ }^{1}$ \\ H. J. Kullmann ${ }^{3}$ \\ L. Freitag'
}

\author{
Survival and Pattern of Failure in Palliative Endobronchial HDR - Brachytherapy \\ Using Iridium 192 in Recurring Bronchial Carcinoma. A Matched Pair Study
}

\section{Zusammenfassung}

Zur Ermittlung der Todesursache und der Überlebenszeit nach palliativer endobronchialer Brachytherapie eines nach externer Strahlentherapie rezidivierenden zentralen Bronchialkarzinoms führten wir eine matched pair Studie durch. 94 Patienten mit Tumorrezidiv nach externer Strahlentherapie erhielten eine Brachytherapie. Sie wurden nachverfolgt und verglichen mit einem retrospektiv gematchten Kollektiv von 94 Patienten, die nicht endoluminal bestrahlt worden waren. Verglichen wurden Alter, Geschlecht, Raucherverhalten, Histologie, Tumorstadium sowie externe Strahlendosis und Fraktionierung. Brachytherapiert wurde in den meisten Fällen mit $3 \times 5 \mathrm{~Gy}$ in $10 \mathrm{~mm}$ Abstand von der Strahlenquelle in Wochenabständen. Führende Todesursache in beiden Kollektiven war mit über $30 \%$ die Tumorgeneralisation. Die terminale Massenblutung ist mit 27,7\% in der Brachytherapiegruppe 2,5-mal höher als in der nur extern bestrahlten Gruppe, während die Todesursache respiratorische Insuffizienz hier mit $11,7 \%$ fast doppelt so hoch lag wie in der Brachytherapiegruppe. Eine endobronchiale Komplettremission führte zu einem 10,5 Monate verlängerten Überleben. Die an Hämoptoe verstorbenen Brachytherapiepatienten leben median 10,2 Monate länger als die matched pair-Patienten mit gleicher Todesursache. Als Ursache der massiven Hämoptoe wurde bei etwa $20 \%$ der kombiniert behandelten Patienten aufgrund des frühen Auftretens innerhalb der ersten 10 Monate ein Strahlentherapieschaden als führende Ursache angenommen. Bei den nach dem 10. Monat verstorbenen Patienten ist als Ursache vorwiegend

\section{Abstract}

To evaluate the impact of palliative high dose rate brachytherapy on survival and a pattern of failure, we performed a matched pair study. 94 patients with tumor recurrence after external beam radiation received endobronchial brachytherapy. They were followed prospectively and matched retrospectively with 94 comparable patients who had not received brachytherapy. Matched parameters were age, gender, smoking behaviour, histology, tumor stage, EBRT-dose and fractionation. The leading cause of death in both groups was generalized tumor growth. In the combined therapy group, fatal hemorrage was $27.7 \%$, two and a half times higher than in the EBRT group with $10.6 \%$, whereas respiratory insufficiency in the brachytherapy group was $6.4 \%$ and $11.7 \%$ in the EBRT group. A complete remission after brachytherapy yielded a 10.5 months longer mean survival. Patients dying from fatal hemorrhage after endobronchial brachytherapy lived on average 10.2 months longer than matched EBRT patients dying from the same cause. Analyzing the timecourse of fatal hemorrage in the brachytherapy group we conclude that - because of its early onset in the first 10 months after induction of therapy roughly $20 \%$ of the deaths can be attributed to a radiation damage. In those patients who died after 10 months the major cause of fatal hemorrhage was the natural course of sqamous cell carcinoma with prolonged survival.

${ }^{1}$ Abteilung für Pneumologie, der Lungenklinik Hemer

${ }^{2}$ Abteilung für Strahlentherapie der Lungenklinik Hemer

${ }^{3}$ von-Huyssen-Stiftung Essen, Pneumologische Abteilung

Korrespondenzadresse

Priv.-Doz. Dr. H.-N. Macha · Pneumologische Abteilung · Lungenklinik Hemer ·

58675 Hemer · E-mail: pneumo@lungenklinik-hemer.de

Eingang: 23. Juli 2004 - Nach Revision alkzeptiert: 16. September 2004

Bibliografie

Pneumologie 2005; 59: 12-17 @ Georg Thieme Verlag KG Stuttgart · New York

DOI 10.1055/s-2004-830138

ISSN 0934-8387 
der durch das verlängerte Überleben begünstigte natürliche Verlauf des Plattenepithelkarzinoms, der zu einer Arrosion der Gefäße führt.

$\longrightarrow$

\section{Einleitung}

Die endobronchiale palliative Brachytherapie ist die älteste interventionelle bronchologische Methode [1]. Mit dem Fortschritt in der Isotopentechnik und der Bronchologie hat sie heute eine weltweite Verbreitung erreicht. Strahlentherapeutisch etabliert ist die High-dose-rate-brachytherapy mit Iridium 192 und der Remote-Afterloading-Technik. Der Applikationskatheter wird in der Regel mittels flexibler Bronchoskopie in Lokalanästhesie positioniert. In einer Umfrage von Nag [2] wird die endobronchiale Brachytherapie allein in den USA an 344 Einrichtungen durchgeführt. Es gibt zahlreiche Publikationen vom klinischen Stellenwert der palliativen endobronchialen Brachytherapie, wobei in der Regel Ansprechraten zwischen 70-90\% angegeben werden (Tab. 1).

Will man die Ergebnisse vergleichen, so stößt man auf erhebliche Handicaps: Unterschiedliche Dosierung, Fraktionierung, Definition der Isodose, fehlende Angaben über Applikatorschläuche, CT-Stadiierung, endoskopischen Befund oder Funktionsparameter erschweren den Vergleich. In besonderen Misskredit gelangte die palliative endobronchiale Brachytherapie durch eine erschreckend hohe Rate terminaler Blutungen, die zwischen 20 und 30\%, in kleineren Kollektiven bis zu 50\% der behandelten Patienten reicht (Tab. 2 ).

Prospektive randomisierte Studien zur Klärung dieser wichtigen Frage gibt es nicht. Wie schwierig es ist, an einem Kollektiv schwer kranker Patienten valide Daten zu ermitteln, zeigt das Scheitern einer prospektiv randomisierten Studie in England [3], in der die Effizienz einer alleinigen palliativen endobronchialen Brachytherapie gegenüber alleiniger externer Strahlentherapie geprüft werden sollte. Um eine 15\%ige Verbesserung der Dyspnoerate über 4 Monate zu beweisen, sollten 400 Patienten eingeschlossen werden. Die Studie wurde nach $3 \frac{1}{2}$ Jahren mit nur 75 eingeschlossenen Patienten abgebrochen, obwohl alle Beteiligten sich über die Wichtigkeit der Fragestellung einig waren.

In der Lungenklinik Hemer ist die endobronchiale Brachytherapie seit 1987 ein etabliertes interventionelles Verfahren. Auch wir konnten im follow up dieser Patienten bei palliativer Indikation eine beunruhigend hohe Rate terminaler Blutungen beobachten, die zwischen 20 und 30\% zu liegen schien. Wir sahen hier dringenden Klärungsbedarf, aber angesichts der Schwierigkeiten, eine randomisierte prospektive Studie an schwerstkranken Patienten durchzuführen, wählten wir stattdessen das Instrument des matched-pair-Vergleiches. Das Verfahren benutzt zwar teilweise retrospektiv gewonnene Daten, und ein Selektions Bias kann nicht ganz vermieden werden, es liefert aber wissenschaftlich verwertbare Ergebnisse mit dem Evidenzgrad B. Schon früher konnten wir dieses Verfahren bei der Evaluierung der endobronchialen Laserrekanalisation erfolgreich einsetzen [4]. Ziel dieser Untersuchung war die Ermittlung der Hämoptoe-
Tab. 1 Ansprechraten nach palliativer endobronchialer Brachytherapie

\begin{tabular}{|lllll}
\hline Autor & $\begin{array}{l}\text { Radiologischer } \\
\text { Befund \% }\end{array}$ & $\begin{array}{l}\text { Endoskopischer } \\
\text { Befund \% }\end{array}$ & $\begin{array}{l}\text { Klinik } \\
\%\end{array}$ & $\begin{array}{l}\text { Pat. } \\
\text { Anzahl }\end{array}$ \\
\hline Speiser [5] & 70 & 80 & 99 & 295 \\
\hline Bedwinek [6] & 64 & 82 & 76 & 32 \\
\hline Gauwitz [7] & 83 & 100 & 88 & 24 \\
\hline Taulelle [8] & 54 & 54 & 74 & 189 \\
\hline Macha [9] & 88 & 75 & 74 & 365 \\
\hline Muto [10] & 90 & - & 90 & 320 \\
\hline Celebioglu [11] & 90 & 85 & 85 & 95 \\
\hline Gollins [12] & 46 & - & 92 & 406 \\
\hline
\end{tabular}

Tab. 2 Häufigkeit terminaler Hämoptoe nach palliativer endobronchialer Brachytherapie

\begin{tabular}{|lccc}
\hline Autor & Dose-rate & \% Hämoptoe & Pat.Anzahl \\
\hline Speiser [5] & HDR & 8,0 & 295 \\
\hline Gollins [12] & HDR & 7,9 & 390 \\
\hline Macha [9] & HDR & 21,0 & 365 \\
\hline Sutedja [20] & HDR & 32,0 & 31 \\
\hline Bedwinek [6] & HDR & 32,0 & 38 \\
\hline Schedel [13] & HDR & 41,0 & 50 \\
\hline Kanavkar [14] & HDR & 50,0 & 12 \\
\hline
\end{tabular}

rate bei kombiniert intern und extern palliativ bestrahlten Patienten im Vergleich zu nur extern bestrahlten Patienten, der Einfluss der Brachytherapie auf andere Todesumstände und auf das Überleben in Abhängigkeit vom endobronchial erreichten Remissionsgrad.

\section{Material und Methode}

Zwischen 1989 und 1995 wurden in der Lungenklinik Hemer 94 Patienten mit einem Bronchialkarzinom einer externen Strahlentherapie unterzogen und anschließend wegen eines lokalen Rezidivs mit einer endobronchialen Brachytherapie mit Iridium 192 high dose rate behandelt. Durchschnittlich wurden 2,9 Brachytherapien pro Patient durchgeführt, die mediane Gesamtdosis betrug 15,36 Gy in $10 \mathrm{~mm}$ Abstand von der Strahlenquellenachse, die mediane bestrahlte Strecke betrug $50 \mathrm{~mm}$. Die Patienten wurden im Rahmen der Tumornachsorge prospektiv untersucht. Zu diesen 94 Patienten gelang es, retrospektiv matched pairs zu bilden, die hinsichtlich Alter, Geschlecht, Tumorstadium, Histologie, Raucherverhalten, externer Strahlentherapie und Fraktionierung gleich waren und diese sich somit nur in einem Punkt vom Vergleichskollektiv unterschieden, nämlich dass sie keine Brachytherapie erhielten (Tab. 3). Mit Hilfe eines Fragebogens des Tumornachsorgeregisters der Lungenklinik Hemer wurden Überleben und Todesumstände dieser Patienten retrospektiv ermittelt und verglichen. 
Tab. 3 Palliative endobronchiale Brachytherapie. Verglichene Parameter im matched-pair-Verfahren

\begin{tabular}{|lll|}
\hline Patienten & $\begin{array}{l}\text { nur extern bestrahlte } \\
\text { extern + brachy- } \\
\text { therapierte Patienten }\end{array}$ \\
\hline n & 94 & 94 \\
\hline Alter & 60,8 & 60,8 \\
\hline männlich & 88 & 88 \\
\hline weiblich & 6 & 6 \\
\hline Raucher \% & 86 & 86 \\
\hline Plattenepithel-Ca \% & 84 & 86 \\
\hline Adeno-Ca \% & 9 & 8 \\
\hline großzelliges Ca \% & 7 & 6 \\
\hline Stadium I + II & 13 & 13 \\
\hline III A & 25 & 25 \\
\hline III B & 45 & 45 \\
\hline IV & 11 & 11 \\
\hline externe Strahlendosis & 57,6 Gy & 53,9 Gy \\
\hline Fraktionierung & $5 \times 2,0$ Gy & $5 \times 2,0$ Gy \\
\hline Brachytherapiedosis & 0 Gy & 15,36 Gy \\
\hline
\end{tabular}

Die statistische Auswertung der Überlebenszeit erfolgte nach der Methode von Kaplan und Meier, die Signifikanzberechnung unter Verwendung des Logrank-Testes mit einem niedrigsten Signifikanzniveau von $\mathrm{p}<0,05$.

\section{Ergebnisse}

\section{Überleben in Abhängigkeit von der Brachytherapie}

Die Überlebenskurve der beiden gematchten Vergleichskollektive mit jeweils 94 Patienten ab dem Zeitpunkt der histologischen Erstdiagnose zeigt im Logrank-Test keinen statistischen signifikanten Unterschied (Abb.1). Einen nahezu identischen Kurvenverlauf zeigen die Patienten mit einem Plattenepithelkarzinom, die $86 \%$ bzw. $84 \%$ der beiden Kollektive ausmachen. Die mediane Überlebenszeit nach histologischer Diagnose betrug hier 463 Tage und die Ein- und Zweijahresüberlebenszeiten betrugen $86,8 \%$ und $26,7 \%$ für das kombiniert behandelte Kollektiv, im Vergleich zu 269 Tagen und 46,9\% respektive $25,1 \%$ für das nur extern bestrahlte Patientenkollektiv. Der Anteil zensierter Daten beträgt $13,6 \%$ sowie $27,7 \%$. Auch hier wird das Signifikanzniveau nicht erreicht.

Betrachtet man aber den durch Brachytherapie endoskopisch diagnostizierten Remissionsgrad und seinen Einfluss auf das Überleben, so fallen die Ergebnisse eindeutig aus. Bei 10 Patienten konnte endoskopisch-histologisch eine Komplettremission erreicht werden. Diese 10 Patienten leben mit median 429 Tagen 302 Tage länger als 54 Patienten, die nur eine PR, MR oder NC erreichen konnten (median 126,5 Tage). Der Unterschied ist mit $\mathrm{p}<0,01$ signifikant. Die Ein- und Zweijahresüberlebensraten betragen für die CR-Patienten $77,8 \%$ und $11,1 \%$, respektive $17,6 \%$ und 2,68\% für die PR, MR und NC-Gruppe (Abb. 2).

Bei den Todesursachen führt mit 30,8\% respektive 31,9\% in beiden Gruppen die Tumorgeneralisierung (Tab.4). In der kombi-

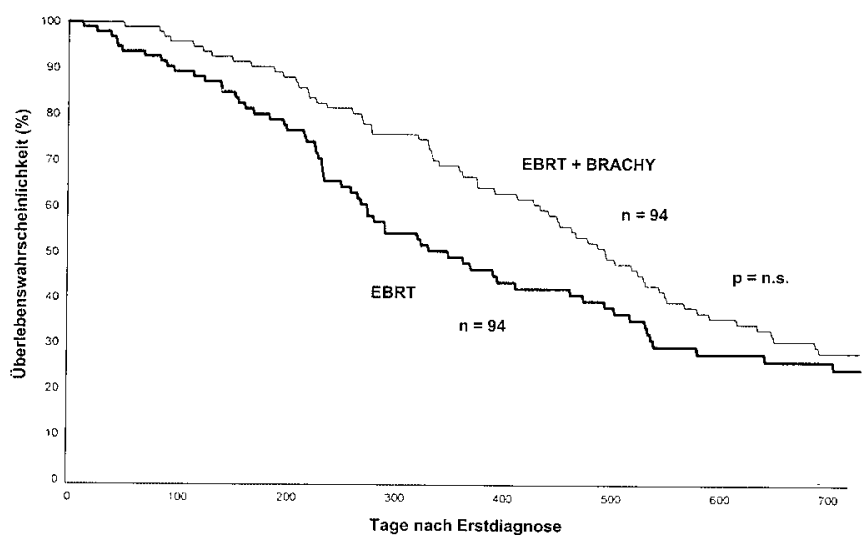

Abb. 1 Endobronchiale HDR-Brachytherapie. Überlebenskurven von 94 Patienten mit externer Strahlentherapie (EBRT) und 94 Patienten mit der Kombination von endobronchialer Brachytherapie + EBRT ab dem Zeitpunkt der Erstdiagnose ( $p=$ n. s.).

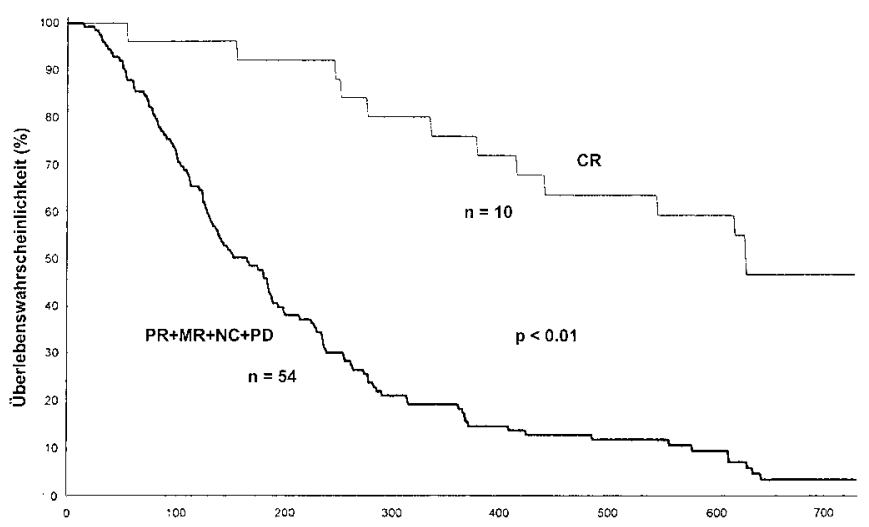

Abb. 2 Überlebenskurven ab Beginn der Brachytherapiebehandlung. Vergleich von 10 Patienten mit endobronchialer Komplettremission (CR) mit 54 Patienten mit PR, MR und NC $(p<0,01)$.

niert behandelten Gruppe ist mit 27,7\% die terminale Hämoptoe $2^{1} / 2$ mal so häufig wie in der Gruppe der externen bestrahlten Patienten. Bei diesen ist aber mit 10,6\% die Todesursache respiratorische Insuffizienz fast doppelt so hoch wie in der kombiniert behandelten Gruppe. Einmalig ist eine tracheobronchiale Fistel als Todesursache aufgetreten, am ehesten als Folge einer extrem hohen Strahlendosis. Als wesentlicher Risikofaktor für das Auftreten einer terminalen Blutung wurde das Plattenepithelkarzinom ermittelt. 23 der 26 an Blutung verstorbenen kombiniert behandelten Patienten $(88,5 \%)$ hatten ein Plattenepithelkarzinom, während alle 10 im extern bestrahlten Kollektiv verstorbenen Patienten an einem Plattenepithelkarzinom litten. Ein weiterer Risikofaktor ist das Tumorstadium III B und die Lokalisation in Trachea und rechtem Hauptbronchus. Die entsprechenden Prozentzahlen lauten für beide Gruppen für III B $57,7 \%$ und $60 \%$ sowie $88 \%$ bzw. $80 \%$ für die Lokalisation in Trachea und rechtem Hauptbronchus.

Vergleicht man das Überleben der an terminaler Hämoptoe verstorbenen Patienten, der kombiniert behandelteten und extern bestrahlten Patienten (Abb.3), so wird eine mediane Überlebenszeit von 200 Tagen ab Erstdiagnose der extern bestrahlten Patienten errechnet, die 1- und 2-Jahresüberlebenszeiten betra- 
Tab. 4 Todesumstände der matched pairs

\begin{tabular}{|lcccc}
\hline & \multicolumn{2}{l}{$\begin{array}{l}\text { kombiniert bestrahlte } \\
\text { Patienten }\end{array}$} & \multicolumn{2}{l}{$\begin{array}{l}\text { nur extrem bestrahlte } \\
\text { Patienten }\end{array}$} \\
& $\boldsymbol{n}$ & $\%$ & $\boldsymbol{n}$ & $\%$ \\
\hline Tumorgeneralisierung & 29 & 30,8 & 30 & 31,9 \\
\hline Hämoptoe & 26 & 27,7 & 10 & 10,6 \\
\hline Respirator. Insuff. & 6 & 6,4 & 11 & 11,7 \\
\hline Fistel & 1 & 1,1 & 0 & 0 \\
\hline Hirnmetastasen & 1 & 1,1 & 5 & 5,3 \\
\hline unbekannt & 15 & 15,9 & 7 & 7,5 \\
\hline andere Todesursache & 5 & 5,3 & 6 & 6,4 \\
\hline lebend & 2 & 2,1 & 6 & 6,4 \\
\hline verloren & 9 & 9,6 & 19 & 20,2 \\
\hline gesamt & 94 & 100,0 & 94 & 100,0 \\
\hline
\end{tabular}

gen $30 \%$ und $10 \%$. Die mediane Überlebenszeit der an Hämoptoe verstorbenen 26 kombiniert behandelten Patienten beträgt 513 Tage ab Erstdiagnose, die 1- und 2-Jahresüberlebenszeiten sind $76,9 \%$ und 30,8\%. Der Unterschied ist signifikant. Median sterben somit die kombiniert behandelten 10,2 Monate später an terminaler Blutung als die allein extern bestrahlten Patienten.

\section{Diskussion}

Ist das Ziel einer Therapie Heilung, so akzeptieren Patient und Arzt eine hohe Rate von Komplikationen und Risiken. Sie sind auch zu einer Teilnahme an prospektiv randomisierten Studien bereit. Bleibt bei fortgeschrittenen Tumorstadien aber nur die Palliation, so ist es wichtig, den Einfluss einer Therapie auf Morbidität, Überleben und Todesumstände zu kennen. Dies gilt insbesondere für interventionelle endoskopische Eingriffe, die an schwer kranken Patienten mit naturgemäß hohem Risiko vorgenommen werden. Hier gibt es immer noch zu wenige Daten über Langzeitmorbidität und Todesumstände. Sie sind allerdings auch schwierig zu ermitteln. Wir konnten mit dem matched-pair-Verfahren an 150 Patienten nachweisen, dass palliativ endobronchial mittels Neodym-YAG-Laser erfolgreich rekanalisierte Patienten nicht nur funktionell profitieren, sondern auch median 4 Monate länger leben, allerdings um den Preis einer sehr hohen Rate terminaler Blutungen von 34,5\% [4]. Auch hier war ein besonderer Risikofaktor der Zelltyp Plattenepithelkarzinom. Die Ergebnisse der vorliegenden Arbeit zeigen einige Verwandtschaft mit der erwähnten Arbeit, was nicht verwundert, da es ein Patientengut mit gleichartigen Risiken ist.

Betrachtet man das Langzeitüberleben vom Zeitpunkt der histologischen Erstdiagnose an, so zeigt das kombiniert behandelte Patientenkollektiv zwar einen tendenziell günstigeren Verlauf, die Daten erreichen aber keine statistische Signifikanz. Realistisch betrachtet ist auch nicht zu erwarten, dass ein nur lokal wirksames Therapieverfahren (die tumorreduzierende Dosis bei Brachytherapie entspricht etwa dem Volumen eines Zeigefingers) Auswirkungen auf ein schon fortgeschrittenes Tumorstadium (III A, III B oder IV) haben kann. In der randomisiert prospektiv durchgeführten Studie von Huber u. Mitarb. [15], die unter

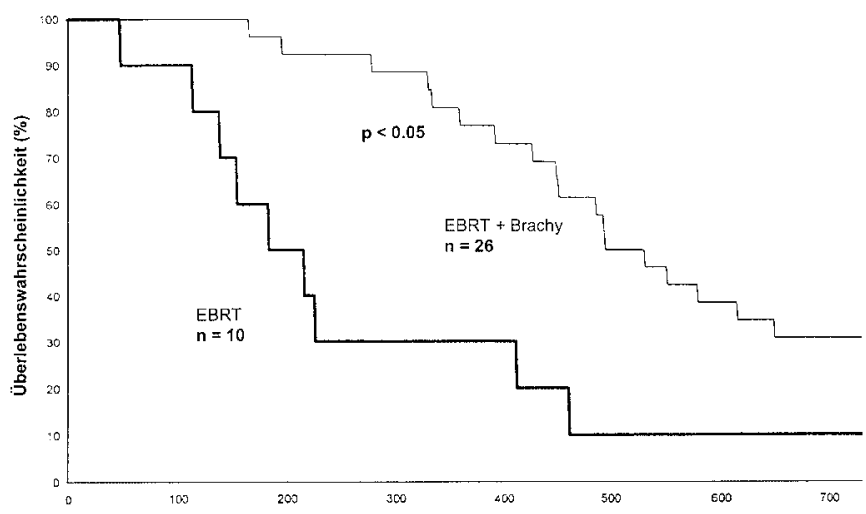

Abb. 3 An terminaler Hämoptoe verstorbene Patienten. Vergleich von EBRT und Kombination von endobronchialer Brachytherapie + EBRT $(p<0,05)$.

kurativem Aspekt externe Strahlentherapie mit der Kombination einer Brachytherapie-Boost-Bestrahlung untersuchte, konnte ebenfalls kein Überlebensvorteil des kombiniert behandelten Gesamtkollektives ermittelt werden. Bei der Untergruppe von Patienten mit einem Plattenepithelkarzinom war ein marginaler Überlebensvorteil zu erkennen. Die untersuchten Patientengruppen waren mit 53 respektive 39 allerdings relativ klein.

Gelingt es aber, mittels Brachytherapie eine histologisch abgesicherte Komplettremission zu erreichen, so ist die Überlebenszeit signifikant und klinisch bedeutsam verlängert: Vergleicht man bei den brachytherapierten Patienten diejenigen mit einer Komplettremission mit denen ohne vorherige Komplettremission, findet sich eine mediane Lebensverlängerung von 10,5 Monaten. Dies ist sehr viel länger als in der vergleichbaren Studie mit Lasertherapie. Hier betrug der Überlebensvorteil nur 4 Monate [4]. Dies erklärt sich am ehesten durch die höhere Tiefenwirkung der Brachytherapie im Vergleich zur Laserkoagulation. Die neueren Methoden zur Bestimmung der Tumorausdehnung in allen Dimensionen wie das hochauflösende $\mathrm{CT}$, die Autofluoreszenzbronchoskopie und endobronchialer Ultraschall standen zu Beginn dieser Studie noch nicht zur Verfügung.

Dass eine alleinige Brachytherapie bei limitiertem Tumorwachstum einen beachtlichen Einfluss auf das Überleben haben kann, zeigte Marsiglia in einer Pilotstudie an 34 Patienten mit inoperablem Bronchialkarzinom - allerdings nur unter Einsatz der CT -, hier wird eine 2-Jahres-Überlebensrate von 78\% angegeben [16]. Die Kombination von kurativer Brachytherapie und photodynamischer Therapie ist bei strikt begrenztem Tumorwachstum (Autofluoreszenz und CT) noch effektiver: In einer Pilotstudie bei 32 Patienten konnte eine Komplettremission von 75\% und ein 2jähriges Überleben von 100\% erreicht werden [17]. Die Patienten in der hier vorgestellten Studie litten an fortgeschrittenem Tumorleiden, die Bestrahlung folge in palliativer Absicht. Nach 2 Jahren lebte nur noch ein einziger brachytherapierter Patient.

Die Analyse der Patienten, die an einer fulminanten Hämoptoe verstorben sind, ergibt nach Brachytherapie einen sehr hohen Anteil von 27,7\%. Der Verlauf der Kaplan-Meier-Kurve der Verstorbenen zeigt, dass sich die Häufigkeit der Blutungen nahezu gleichmäßig über einen Zeitraum von fast 2 Jahren verteilte, 
noch nach 650 Tagen verstarb ein Patient an einer fulminanten Blutung.

Grundsätzlich ist die endobronchiale Brachytherapie ein einfaches und sicheres Verfahren. Die akute Komplikationsrate entspricht der einer fiberoptischen Bronchoskopie. Speiser und Spratling [18] beschrieben in einem großen Kollektiv in $12 \%$ der Patienten eine Strahlenreaktion an der Bronchialschleimhaut. Sie unterschieden eine Frühform etwa bis 19 Wochen nach erster Brachytherapie mit Schwellung und Rötung, aber folgenloser Abheilung und einer Spätreaktion, die weitaus ernster zu bewerten ist. Hier kommt es zu Schleimhautulzerationen, Knorpelnekrosen und später narbigen Strikturen, die wiederum andere interventionelle bronchologische Maßnahmen erfordern. Der Zeitpunkt dieser Schäden liegt bei 43 - 45 Wochen nach erster Strahlentherapie und entspricht damit der bekannten klassischen Spätreaktion. In dieser Gruppe beobachteten Speiser und Spratling eine $25 \%$ ige Rate von terminaler Hämoptoe. In dem von uns untersuchten Patientenkollektiv, die an terminaler Hämoptoe verstorben waren, lag bei 5 Patienten der Todeszeitpunkt vor der 45. Woche. Huber u. Mitarb. [15] beobachteten in ihrem randomisierten prospektiven Patientenkollektiv ebenfalls eine Blutungsrate von $20,8 \%$ ( 11 von 53 ) bei der kombiniert behandelten Gruppe gegenüber 15,3 (6 aus 39) in der extern bestrahlten Gruppe, geben aber den Todeszeitpunkt nicht an.

Bei der Bewertung der relativ hohen Rate terminaler Blutungen muss zwischen den Direkttherapiefolgen und dem natürlichen Verlauf des Bronchialkarzinoms differenziert werden. Eine Hämoptoe als Therapiefolge nach Brachytherapie tritt in der Regel wie nach externer Strahlentherapie - zwischen 1 und 10,5 Monaten auf. Die Wünsche des Endoskopikers widersprechen oft der Strahlenphysik. Das Hauptrisiko für Lokalkomplikationen ist die Verwendung zu dünner Applikatorsonden. Diese sind zwar einfach durch den Arbeitskanal des flexiblen Bronchoskopes zu platzieren, haben aber entscheidende Nachteile: In Trachea, Haupt- und Lappenbronchien liegen sie immer exzentrisch und haben an einigen Stellen direkten Schleimhautkontakt. Verwendet man die Sonden mit Außendurchmessern von $1,8 \mathrm{~mm}$, wie sie von den Markführern Sauerwein und Selectron vertrieben werden, ergibt sich eine Schleimhautkontaktdosis von $90 \mathrm{~Gy}$. Im Bereich dieser „hot spots“ kommt es regelmäßig zu Schleimhautund Knorpelnekrosen des gesunden Gewebes. Kontaktbestrahltes Tumorgewebe schmilzt in kürzester Zeit ein, und es kann massiv bluten. Schon die Verwendung eines Applikators mit $4 \mathrm{~mm}$ Außendurchmesser reduziert die Schleimhautkontaktdosis auf 32 Gy mit sehr viel geringeren Auswirkungen. Das Ziel, eine möglichst gleichförmige Isodose am Tumor zu erreichen und eine exzentrische Lage des Brachytherapiekatheters zu vermeiden, kann bei Tumorstenosen durch eine vorherige Laserabtragung erreicht werden. Nach endoluminaler Tumorverkleinerung kann man beispielsweise anstelle eines $1,8 \mathrm{~mm}$ Katheters einen $4 \mathrm{~mm}$ Applikator einlegen. Im Bereich von Trachea und Hauptbronchien mit weitem Lumen empfiehlt sich die Anwendung eines Spreizkorbapplikators, der zu einer homogeneren Dosisverteilung führt [19].

Während die terminale Hämoptoe vor dem 10. Monat nach Brachytherapie auf eine therapieinduzierte Komplikation hinweist, ist die spätere Hämoptoe eher als natürlicher Verlauf des Plat- tenepithelkarzinoms zu deuten. Durch eine erfolgreiche endobronchiale Intervention lebt der Patient lange genug, so dass der Tumor nun auch die Gefäße arrodieren kann. Betrachtet man unter diesem Aspekt die minimalen und maximalen Überlebenszeiten der an terminaler Hämoptoe verstorbenen Patienten in der Literatur, soweit sie mitgeteilt werden, so liegen sie bei Kanavkar zwischen 14 und 217 Tagen [14], Bedwinek [6] zwischen 14 und 392 Tagen, Sutedja [20] zwischen 14 und 168 Tagen und Seagrean [21] zwischen 120 und 870 Tagen, also sehr früh.

Unter diesem zeitlichen Aspekt betrachtet, kommen in unserem matched-pair-Vergleich nur 5 von 26 kombiniert behandelten Patienten innerhalb von 315 Tagen durch Hämoptoe zu Tode gegenüber 7 von 10 in der nur extern bestrahlten Gruppe. Alle hatten ein Plattenepithelkarzinom. Die führende Todesursache beider Kollektive ist die Tumorgeneralisation. In der kombiniert behandelten Gruppe folgt mit 27,7\% die terminale Blutung und die respiratorische Insuffizienz mit 6,4\%. In der matched-pair-Gruppe nach nur externer Strahlentherapie steht an zweiter Stelle mit $11,7 \%$ die respiratorische Insuffizienz und ist damit doppelt so hoch wie in der kombiniert behandelten Gruppe. Eine erfolgreiche endobronchiale Intervention beeinflusst somit auch hier wie beim Laser [4] die Todesumstände. Erst an dritter Stelle in der extern bestrahlten Gruppe kommt mit 10,6\% die massive Blutung.

Eine gewisse Unsicherheit in der Bewertung der Todesumstände ist die mit 20,2 \% relativ große Gruppe von Patienten im Kontrollarm, die im follow up verlorengegangen sind. Dies ist die immanente Schwäche eines retrospektiv untersuchten Kollektivs und muss aus den eingangs erörterten Gründen in Kauf genommen werden. Dennoch folgern wir, dass bei palliativer endobronchialer Brachytherapie in dem von uns untersuchten Patientengut in knapp 20\% eine letale Blutung als Strahlentherapiefolge wahrscheinlich ist. Durch Optimierung der Applikatorsonden und der Strahlendosis ist hier eine Verbesserung möglich. In etwa $80 \%$ der an einer Massenblutung verstorbenen Patienten wird durch die Brachytherapie das Überleben der Patienten verlängert, und die terminale Hämoptoe ist im Verlauf des Bronchialkarzinoms begründet.

\section{Literatur}

${ }^{1}$ Yankauer S. Two cases of lung tumour treated bronchoscopically. New York Med J 1922; 21: 741 - 742

${ }^{2}$ Nag S, Owen JB, Farnan N et al. Survey of Brachytherapy Practice in the United States: A report of the Clinical Research Comittee of the Amerian Endocurie therapy Society. Int J Rad Oncol Biol Phys 1995; 31: $103-107$

${ }^{3}$ Moghissi K, Boud HG, Sambrock RJ et al. Treatment of endotracheal or endobronchial obstruction by non-small cell lung cancer: Lack of patient in an MRC randomized trial leaves key questions unanswered Medical Research Council Lung Cancer working party. Clin Oncol (R Coll Radiol) 1999; 11 (3): 179-193

${ }^{4}$ Macha HN, Becker KO, Kemmer HP. Pattern of failure an survival in endobronchial laser resection. A matched pair study. Chest 1994; 105: $1668-1672$

${ }^{5}$ Speiser BL, Spratling L. Remote afterloading brachytherapy for local control of endobronchial carcinoma. Int J Radiol Oncol Biol Phys 1993; 25: $579-587$

${ }^{6}$ Bedwinek J, Petty A, Burton C et al. The use of high dose rate endobronchial brachytherapy to palliate symptomatic endobronchial re- 
currence of previously irradiated bronchogenic carcinoma. Int J Radiol Oncol Briol Phys 1991; 22: 23 - 30

${ }^{7}$ Gauwitz M, Ellerbrock A, Komaki N. High dose rate endobronchial irradiation in recurrent bronchial carcinoma. Chest 1985; 88: 810-814

${ }^{8}$ Taulelle M, Chauret B, Vincent P et al. High dose rate endobronchial brachytherapy: results and complications in 189 patients. Eur Resp J 1998; 11 (1): $162-168$

${ }^{9}$ Macha HN, Wahlers B, Reichle G et al. Endobronchial radion therapy or obstructing malignancies: ten years experience with Iridium 192 high dose radiation (HDR) brachytherapy afterloading technique in 365 patients. Lung 1995; 1973: $271-280$

${ }^{10}$ Muto P, Ravo V, Panellit G et al. High dose rate brachytherapy of bronchial cancer: treatment optimisation using three schemes of therapy. Oncologise 2000; 5 (3): 209-214

${ }^{11}$ Celebioglu B, Gurkan OU, Erdogan S. High dose rate endobronchial brachytherapy effectively palliates symptoms due to inoperable lung cancer. J Clin Oncol 2002 Nov; 32 (11): 442 - 448

${ }^{12}$ Gollins SW, Burt PA, Barber PV et al. High dose rate intraluminal radiotherapy for carcinoma of the bronchus: outcome of treatment in 406 patients. Radiotherapy and Oncology 1994; 33: $31-40$

${ }^{13}$ Schedel H, Rohloff R, Huber RM et al. Endoluminale Bestrahlung mit dem HDR-Iridium 192 Afterloadingverfahren bei tracheobronchialen Tumoren. Strahlenther Oncol 1988; 165: 202 - 207
${ }^{14}$ Kanavkar BP, Stern B, Albeti W et al. Complications associated with brachytherapy alone or with laser in lung cancer. Chest 1991; 99: $1062-1065$

${ }^{15}$ Huber RM, Pischer R, Plllinger B et al. Does additional brachytherapy improve the effect of external radiation? A prospective randomized study in central lung tumors. Eur Resp J 1996; 9 Suppl 23: 105

${ }^{16}$ Marsiglia H, Baldegrou P, Lartigau E et al. High dose rate brachytherapy as sole mordality for early-stage endobronchial carcinoma. Int J Radiol Oncol Biol Phys 2000 Jun 1; 47 (3): 665-672

${ }^{17}$ Freitag L, Ernst A, Thomas M et al. Improved local tumor control of limited bronchogenic carcinoma using sequential photodynamic therapy and high dose brachytherapy. Thorax 2003, in press

${ }^{18}$ Speiser BL, Spratling L. Radiation bronchitis and stenosis secondary to high dose rate endobronchial irradiation. Int J Radiat Oncol Biol Phys 1993; 25: $589-597$

${ }^{19}$ Fritz P, Schraube P, Becker HD et al. A new Applicator, positionable to the Center of tracheobronchial lumen for HDR-IR192 Afterloading of tracheobronchial tumors. Int J Radiol Oncol Biol Phys 1992; 20: $1061-1066$

20 Sutedja T, Baris G, Zoetmulder F et al. High dose Rate Brachytherapy in Patients with Local Recurrences after Radiotherapy of Non-Small cell lung cancer. Int J Radiol Oncol Biol Phys 1992; 24: 551 - 552

21 Seagren StL, Harrel JH, Horn RA. High dose Rate Intraluminal Irradiation in Recurrent Endobronchial Carcinoma. Chest 1985; 88: 810-814 